\title{
Schriftliche Fragen
}

mit den in der Woche vom 15. September 2008

eingegangenen Antworten der Bundesregierung

\section{Verzeichnis der Fragenden}

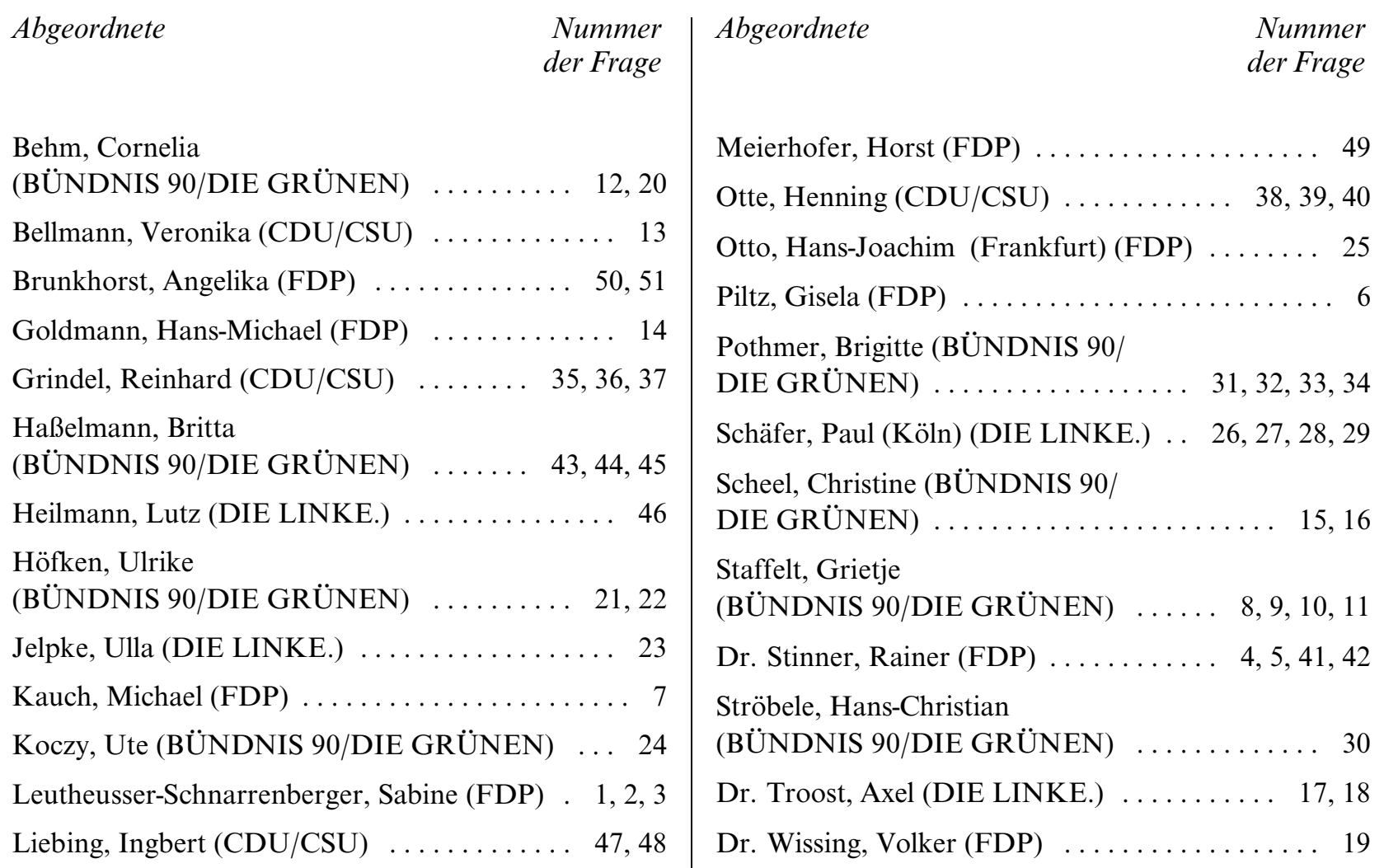




\section{Verzeichnis der Fragen nach Geschäftsbereichen der Bundesregierung}

\begin{abstract}
Seite

\section{Geschäftsbereich des Auswärtigen Amts}

Leutheusser-Schnarrenberger, Sabine (FDP) Maßnahmen der Bundesregierung auf nationaler, EU- und UN-Ebene in Bezug auf das Listungsverfahren von Terrorverdächtigen zugunsten der Rechte Betroffener nach dem entsprechenden Urteil des EuGH vom 3. September 2008 . . . . . . . . . . . . . . 1
\end{abstract}

Dr. Stinner, Rainer (FDP)

Einseitige Kündbarkeit der deutschen Zusagen für Finanzhilfen an Russland im Rahmen der G8-Initiative Globale Partnerschaft gegen die Weiterverbreitung von Massenvernichtungswaffen; Gründe für die nicht mögliche Finanzierung dieser Projekte allein aus russischen Mitteln

\section{Geschäftsbereich des Bundesministeriums des Innern}

Piltz, Gisela (FDP)

Abfrage von Daten zu Bürgern über Internetdienste wie „yasni“ oder „Xing“ durch die Bundesbehörden ................ 3

\section{Geschäftsbereich des Bundesministeriums der Justiz}

Kauch, Michael (FDP)

Stand und Vorlage des im Auftrag des Bundesministeriums der Justiz durchgeführten Forschungsprojektes „Kinder in gleichgeschlechtlichen Lebensgemeinschaften“ . . . . 3
Seite

Staffelt, Grietje

(BÜNDNIS 90/DIE GRÜNEN)

Kenntnis der Bundesregierung zum Stand und Inhalt der Verhandlungen über ein multinationales Abkommen zur Durchsetzung von geistigen Eigentumsrechten, dem Anti-Counterfeiting Trade Agreement (ACTA), sowie Beteiligung und Position der Bundesregierung; geplante deutsche Initiativen bei Urheberrechtsverletzungen im Internet unter Berücksichtigung des französischen Modells; geplante weitere Reform des Urheberrechts in dieser Legislaturperiode

\section{Geschäftsbereich des Bundesministeriums der Finanzen}

Behm, Cornelia

(BÜNDNIS 90/DIE GRÜNEN)

Haltung der Bundesregierung zur Genehmigung der Mineralölsteuerermäßigung für die französische Landwirtschaft im Vergleich zur Entscheidung der EU-Kommission zur deutschen Mineralölsteuerrückerstattung für Unterglasgartenbaubetriebe $\quad$. 6

Bellmann, Veronika (CDU/CSU)

Haltung der Bundesregierung zur geplanten einheitlichen Besteuerung des Elterngeldes auf Länderebene mit einer damit verbundenen Aufhebung der sächsischen Sonderregelung eines steuerfreien Sockelbetrags .... 7

Goldmann, Hans-Michael (FDP) Festhalten der Bundesregierung an der Anweisung zur Versteuerung von zwei Betriebsprämien bei Landwirtschaftsbetrieben für das zu Ende gegangene Wirtschaftsjahr $2007 / 2008$. . . . . . . . . . . . . . . . . 7 
Scheel, Christine

(BÜNDNIS 90/DIE GRÜNEN)

Zugrunde liegende steuerliche Maßnahmen sowie Steuerausfälle für die erwähnte Energiesteuerbefreiung beim Flugbenzin von ca. 7 Mrd. Euro auf S. 13 der Veröffentlichung des Bundesministeriums für Umwelt, Naturschutz und Reaktorsicherheit „Ökologische Industriepolitik - nachhaltige Politik für Innovation, Wachstum und Beschäftigung“ .................. 8

Dr. Troost, Axel (DIE LINKE.)

Wirtschaftliche und politische Ziele durch das Angebot der Tagesanleihe der Finanzagentur seit dem 1. Juli 2008 sowie mögliche Gefahren für Sparkassen und Genossenschaftsbanken durch dieses neue Finanzprodukt im Privatkundenbereich ........ 9

Dr. Wissing, Volker (FDP)

Anzahl und Inhalt der Gesetzesänderungen zur Besteuerung von betrieblich genutzten Personenkraftwagen seit Beginn der 14. Legislaturperiode ................

\section{Geschäftsbereich des Bundesministeriums für Wirtschaft und Technologie}

Behm, Cornelia

\section{(BÜNDNIS 90/DIE GRÜNEN)}

Handlungsmöglichkeiten der Bundesregierung gegen etwaige Preisabsprachen der Produzenten von Düngemitteln in Anbetracht des massiven Preisanstiegs innerhalb der letzten Jahre

Höfken, Ulrike

\section{(BÜNDNIS 90/DIE GRÜNEN)}

Inhalte und Ergebnisse des Besuchs einer Delegation des Bundesministeriums für Wirtschaft und Technologie am 8. Juli 2008 im französischen Bure sowie Informationen der Bundesregierung über den Stand der Planungen für ein Atommüllendlager in Bure und Möglichkeiten der Endlagerung deutschen Atommülls in Lothringen . . . . . .

Jelpke, Ulla (DIE LINKE.)

Maßnahmen der Bundesregierung zur Aufklärung der Lieferwege der in Besitz des georgischen Militärs geratenen Sturmgewehre des Typs G36 des Heckler \& KochKonzerns .................
Koczy, Ute (BÜNDNIS 90/DIE GRÜNEN)

Aktueller Stand der Umsetzung des IlisuStaudammprojekts und Verfahrensschritte im Falle einer Kündigung der Verträge über die Hermesbürgschaft für dieses Projekt .................. 14

Otto, Hans-Joachim (Frankfurt) (FDP) Kenntnis und Haltung der Bundesregierung zu Plänen zum Rückbau der Hauptverteiler durch die Deutsche Telekom AG . . . . . . . . 14

Schäfer, Paul (Köln) (DIE LINKE.) Herstellung von G-36-Gewehren und Export nach Georgien ...............

Ströbele, Hans-Christian (BÜNDNIS 90/DIE GRÜNEN)

Maßnahmen der Bundesregierung zur Aufklärung der Herkunft ,deutscher“ G-36-Gewehre in Georgien (vgl. auch die schriftliche Frage 39 auf Bundestagsdrucksache 16/10199) . . . . . . . . . . . . . . . . . . . . 16

\section{Geschäftsbereich des Bundesministeriums für Arbeit und Soziales}

\section{Pothmer, Brigitte}

(BÜNDNIS 90/DIE GRÜNEN)

Voraussichtliche Entsperrung von 1500

Personalstellen der Bundesagentur für Arbeit in den ARGEn und den Agenturen in getrennter Aufgabenwahrnehmung (AAgAw); Abbildung einzelner Tätigkeiten des operativen Geschäfts der ARGEn/ AAgAw in den Mitarbeiterkapazitäten des vorläufigen ganzheitlichen Orientierungsschlüssels sowie entsprechende Größenordnungen laut Ausschussdrucksache 16(11)1068 vom 22. August 2008; dementsprechendes Betreuungsverhältnis unter Einbezug aller erwerbsfähigen Hilfebedürftigen und aller Mitarbeiterkapazitäten im operativen Bereich; Pläne zur Reduzierung der für das Haushaltsjahr 2008 vorgegebenen Obergrenze für befristete Beschäftigungsverhältnisse für BA-Personal in den ARGEn/AAgAw .................. 


\section{Geschäftsbereich des Bundesministeriums der Verteidigung}

Grindel, Reinhard (CDU/CSU)

Konsequenzen der Bundesregierung aus der Veröffentlichung des Plakats „Schritt zur Abrüstung" des Büros für antimilitaristische Maßnahmen (BamM) und des Landesverbands Berlin-Brandenburg der Deutschen Friedensgesellschaft - Vereinigte KriegsdienstgegnerInnen (DFG-VK); öffentliche Gelder für diese Vereinigungen; geplante rechtliche Schritte gegen deren Aktivitäten .......................

Otte, Henning (CDU/CSU)

Konsequenzen der Bundesregierung aus den Aktivitäten des Büros für antimilitaristische Maßnahmen (BamM) und des Landesverbands Berlin-Brandenburg der Deutschen Friedensgesellschaft - Vereinigte KriegsdienstgegnerInnen (DFG-VK), insbesondere aus der Veröffentlichung des Plakats zum Tod von Bundeswehrsoldaten sowie mögliche Erstattung entsprechender Strafanzeigen; öffentliche Gelder für BamM und DFG-VK

Dr. Stinner, Rainer (FDP)

Haltung der Bundesregierung zur Äußerung des Parlamentarischen Staatssekretärs Thomas Kossendey über den Ausschluss der Bekämpfung der Piraterie beim Mandat für die Operation Enduring Freedom am Horn von Afrika . . . . . . . . . . . . . . . . .

Haltung der Bundesregierung zur Zugehörigkeit der Terrorismusbekämpfung zu den deutschen Aufgaben in Afghanistan laut Aussage des Bundesministers der Verteidigung Dr. Franz Josef Jung im heute-journal vom 3. September 2008
Geschäftsbereich des Bundesministeriums für Familie, Senioren, Frauen und Jugend

Haßelmann, Britta

(BÜNDNIS 90/DIE GRÜNEN)

Haltung der Bundesregierung zur Auffassung des Staatssekretärs im Bundesministerium für Familie, Senioren, Frauen und Jugend, Gerd Hoofe, bezüglich einer dauerhaften und abhängigen Förderung von Organisationen und Vereinen im Bereich des bürgerschaftlichen Engagemens . . . . . . . 22

Höhe und Kriterien der Förderung von Organisationen und Vereinen neben den im Kapitel 1702 des Bundeshaushalts 2009 genannten elf institutionell geförderten $\mathrm{Zu}-$ wendungsempfängern und Gründe für die frühzeitige Beendigung der Projektförderung bei einigen Organisationen . . . . . . . 23

\section{Geschäftsbereich des Bundesministeriums für Verkehr, Bau und Stadtentwicklung}

Heilmann, Lutz (DIE LINKE.)

Entscheidung über den möglichen Wegfall von bis zu zwei Schleusen am Elbe-LübeckKanal sowie hierfür erforderliche Anpassungen des Kanalbetts ................. . 25

Liebing, Ingbert (CDU/CSU)

Zeitpunkt der durch die Bundesregierung festgestellten Illegalität der am 12. August 2008 begonnenen Aktion von Greenpeace zur Versenkung von Steinen im Flora-Fauna-Habitat-Gebiet „Sylter Außenriff“" sowie seitdem ergriffene Maßnahmen zur Unterbindung dieser Aktion . . . . . . . . . . . 25

Meierhofer, Horst (FDP)

Pläne für den Wiederaufbau der DonauWehrbrücke (Protzenweiher Brücke) . . . . . 26

Geschäftsbereich des Bundesministeriums für Umwelt, Naturschutz und Reaktorsicherheit

Brunkhorst, Angelika (FDP) Berücksichtigung der Übertragung der finanziellen Verantwortung für die Schachtanlage Asse vom BMBF auf das BMU in den aktuellen Beratungen über den Bundeshaushalt 2009 und etwaige haushälterische Auswirkungen ................. 26 


\section{Geschäftsbereich des Auswärtigen Amts}

1. Abgeordnete

Sabine

Leutheusser-

Schnarrenberger (FDP)

\begin{abstract}
Welche konkreten Maßnahmen ergreift die Bundesregierung nun nach dem Urteil des Europäischen Gerichtshofes vom 3. September 2008 in den Rechtssachen C-402/05 P und C-415/05 P auf nationaler, EU- und UN-Ebene in Bezug auf das Listungsverfahren von Terrorverdächtigen?
\end{abstract}

\section{Antwort des Staatsministers Dr. h. c. Gernot Erler vom 12. September 2008}

Nach der Verkündung des Urteils des Europäischen Gerichtshofes $(\mathrm{EuGH})$ am 3. September 2008 hat der Rat die vom EuGH festgestellten Mängel innerhalb einer Frist von drei Monaten zu heilen. Die französische EU-Ratspräsidentschaft hat bereits die Initiative zu Beratungen in den zuständigen Ratsgremien ergriffen. Die Bundesregierung hat sich dafür eingesetzt, dass in den zuständigen Ratsgremien eine Analyse der Urteilsfolgen und des sich aus dem Urteil ergebenden Handlungsbedarfs erfolgen wird. Sie wird sich an den Beratungen aktiv beteiligen.

2. Abgeordnete

Sabine

Leutheusser-

Schnarrenberger

(FDP)
Wird die Bundesregierung insbesondere initiativ dahingehend werden, dass die ungeprüfte Übernahme der UN-Terrorliste durch die EU beendet wird?

\section{Antwort des Staatsministers Dr. h. c. Gernot Erler vom 12. September 2008}

Die Bundesregierung wird sich bei den Beratungen in den zuständigen EU-Gremien dafür einsetzen, dass das Urteil des EuGH vollständig umgesetzt wird.

3. Abgeordnete

Sabine

Leutheusser-

Schnarrenberger (FDP)
Welche Maßnahmen wird die Bundesregierung ergreifen, damit die von der Listung Betroffenen ein rechtsstaatliches Verfahren, insbesondere die Möglichkeit zur Stellungnahme und Überprüfung der gegen sie erhobenen Vorwürfe, auf nationaler, EU- bzw. UN-Ebene erhalten?

\section{Antwort des Staatsministers Dr. h. c. Gernot Erler vom 12. September 2008}

Die Bundesregierung hat sich schon seit mehreren Jahren gemeinsam mit ihren Partnern in Europa und den Vereinten Nationen für einen Ausbau der rechtsstaatlichen Verfahren im Zuge der Erstellung der 
Sanktionslisten eingesetzt. In Bezug auf die EU-autonomen Listungen nach dem Gemeinsamen Standpunkt 2001/0931/GASP wurden unter deutscher EU-Ratspräsidentschaft erhebliche Verbesserungen eingeführt, so die Unterrichtung der Gelisteten über ihre Listung, die Mitteilung über die Begründung für die Listung, die Möglichkeit zum rechtlichen Gehör und eine Rechtsbehelfsbelehrung. Weiterhin wurde das Ratsgremium, das Listungsfragen nach diesem Gemeinsamen Standpunkt berät, zu einer regulären Ratsarbeitsgruppe umgewandelt.

Auf der Ebene der Vereinten Nationen setzt der Sicherheitsrat in der Regel Sanktionsausschüsse ein, die die verschiedenen Sanktionsregime der Vereinten Nationen begleiten und überprüfen, so auch dasjenige gegen Mitglieder und Unterstützer von Al Qaida und Taliban. In der Regel befinden die Sanktionsausschüsse über die Aufnahme zusätzlicher Personen in Sanktionslisten bzw. über eine mögliche Streichung. Die Bemühungen der Bundesregierung und ihrer Partner zielten in den vergangenen Jahren insbesondere darauf, Methoden zu entwickeln, um diese Verfahren transparenter zu gestalten. Als Ergebnis auch der Bemühungen der Bundesregierung wurde Ende März 2007 eine durch den Sicherheitsrat geschaffene Anlaufstelle geschaffen, bei der Gelistete Streichungsanträge einreichen können.

Die Bundesregierung wird auch bei den Beratungen über eine weitere Stärkung der rechtsstaatlichen Verfahren im Zuge der Umsetzung von VN-Sanktionen durch die EU aktiv mitwirken. Gleichzeitig ist darauf hinzuweisen, dass alle VN-Mitgliedstaaten nach Artikel 25 der VNCharta verpflichtet sind, die Beschlüsse des Sicherheitsrates der Vereinten Nationen anzunehmen und durchzuführen. Das sich hieraus ergebende Spannungsfeld dürfte einen der Schwerpunkte der Beratungen bilden.

4. Abgeordneter Dr. Rainer Stinner (FDP)
Sind die Zusagen für deutsche Finanzhilfen an Russland im Rahmen der G8-Initiative Globale Partnerschaft gegen die Weiterverbreitung von Massenvernichtungswaffen von deutscher Seite aus einseitig kündbar, und wenn ja, beabsichtigt die Bundesregierung, diese Zahlungen trotzdem wie geplant bis 2012 fortzuführen?
5. Abgeordneter Dr. Rainer Stinner (FDP)
Aus welchen Gründen hält es die Bundesregierung für nicht möglich, dass Russland die mit deutscher Unterstützung geplanten Projekte gegen die Weiterverbreitung von Massenvernichtungswaffen und -materialien aus eigenen Mitteln finanziert?

\section{Antwort des Staatsministers Dr. h. c. Gernot Erler vom 12. September 2008}

Auf dem G8-Gipfel in Kananaskis im Jahr 2002 hat die Bundesregierung für Projekte im Rahmen der Globalen Partnerschaft Mittel bis zu einer Obergrenze von 1,5 Mrd. US-Dollar zugesagt. Seit dem G8Gipfel von Heiligendamm besteht Einigkeit, die Globale Partnerschaft 
über die Grenzen der früheren Sowjetunion auszudehnen. Die deutsche Zusage und Obergrenze gelten seither für die Globale Partnerschaft insgesamt, also nicht nur für Projekte in Russland.

Die Zusammenarbeit mit Russland bei der G8-Initiative Globale Partnerschaft besteht aus einzelnen Projekten, für die mit Russland jeweils völkerrechtlich bindende Vereinbarungen abgeschlossen wurden. Mit Durchführungsorganisationen und Unterauftragnehmern wurden rechtlich verbindliche Verträge abgeschlossen. Die Bundesregierung beabsichtigt, diese Verträge einzuhalten.

$\mathrm{Zu}$ weiteren Einzelheiten und zur Begründung der Fortsetzung der Projekte und des deutschen sicherheitspolitischen Interesses an ihnen wird auf die Antwort der Bundesregierung zu Frage 50 der Großen Anfrage der Fraktion BÜNDNIS 90/DIE GRÜNEN vom 26. Juni 2008 (Bundestagsdrucksache 16/9834) verwiesen.

\section{Geschäftsbereich des Bundesministeriums des Innern}

6. Abgeordnete

$$
\text { Gisela }
$$

Piltz

(FDP)
In welchen Fällen werden von Bundesbehörden Details über Internetdienste wie z. B. „yasni“ und „Xing“ zu Bürgerinnen und Bürger (vgl. dazu www.presseportal.de) abgefragt?

\section{Antwort des Parlamentarischen Staatssekretärs Peter Altmaier vom 17. September 2008}

Es gibt keine Fälle, in denen die Bundesregierung und ihr Geschäftsbereich systematisch oder routinemäßig Daten von Bürgerinnen und Bürgern über Personensuchmaschinen oder soziale Netzwerke wie z. B. „yasni“" oder „Xing“ abfragen.

Gelegentlich wird aber zur dienstlichen Aufgabenwahrnehmung und unter Beachtung der geltenden datenschutzrechtlichen Vorgaben auch auf Internetdienste der genannten Art, soweit sie offen (ohne Anmeldung oder Registrierung) zugänglich sind, zugegriffen. Statistische Erhebungen liegen hierzu nicht vor.

\section{Geschäftsbereich des Bundesministeriums der Justiz}

7. Abgeordneter Michael Kauch (FDP)
Wie ist der Sachstand des im Auftrag des Bundesministeriums der Justiz durchgeführten Forschungsprojektes „Kinder in gleichgeschlechtlichen Lebensgemeinschaften", wann ist mit der Veröffentlichung von ersten Ergebnissen sowie der gesamten Studie zu rechnen? 


\section{Antwort des Parlamentarischen Staatssekretärs Alfred Hartenbach vom 15. September 2008}

Das vom Bundesministerium der Justiz (BMJ) beauftragte Forschungsinstitut hat die notwendigen Erhebungen durchgeführt. Es ist derzeit dabei, die Ergebnisse auszuwerten und den Abschlussbericht zu erstellen. Die Veröffentlichung von Teilergebnissen ist nicht vorgesehen. Der Abschlussbericht wird nach Abnahme durch das BMJ in geeigneter Form veröffentlicht. Mit einer Veröffentlichung noch im Jahr 2008 ist nicht zu rechnen.

8. Abgeordnete

Grietje

Staffelt

(BÜNDNIS 90/

DIE GRÜNEN)
Welche Kenntnisse hat die Bundesregierung zu Stand, Inhalt und Zeitplan der Verhandlungen zum Abschluss eines multinationalen Abkommens zur Durchsetzung von geistigen Eigentumsrechten, dem Anti-Counterfeiting Trade Agreement (ACTA), hier auch zu den strafrechtlichen Aspekten des Abkommens, und inwieweit ist die Bundesregierung an diesen Verhandlungen beteiligt?

\section{Antwort des Parlamentarischen Staatssekretärs Alfred Hartenbach vom 15. September 2008}

Bisher haben zwei Verhandlungsrunden, am 3. und 4. Juni 2008 in Genf und vom 29. bis 31. Juli 2008 in Washington D. C. stattgefunden. Für das Jahr 2008 ist eine weitere Verhandlungsrunde geplant. $\mathrm{Zu}$ den Teilnehmern gehören Australien, die Europäische Union, Japan, Kanada, Korea, Mexiko, Marokko, Neuseeland, Singapur, die Schweiz und die USA. Verhandelt wurde bisher über Zollmaßnahmen sowie zivilrechtliche Maßnahmen zur Durchsetzung von Rechten des geistigen Eigentums.

Die EU-Mitgliedstaaten, also auch Deutschland, haben der Europäischen Kommission ein Mandat für die Verhandlungen erteilt, soweit es um Verhandlungsgegenstände geht, für die die Europäische Union zuständig ist. Wenn die Zuständigkeiten der Mitgliedstaaten berührt sind, werden die Mitgliedstaaten in den Verhandlungen durch die jeweilige Ratspräsidentschaft vertreten. Die Bundesregierung nimmt über die zuständigen Gremien auf die Verhandlungsposition der Europäischen Union Einfluss. Sie ist bei den Verhandlungen durch einen Beobachter vertreten.

9. Abgeordnete

Grietje

Staffelt

(BÜNDNIS 90/

DIE GRÜNEN)
Welche Position bezieht die Bundesregierung zu ACTA, und wie begründet sie die für die Öffentlichkeit äußerst intransparenten Verhandlungen?

\section{Antwort des Parlamentarischen Staatssekretärs Alfred Hartenbach vom 15. September 2008}

Die Bundesregierung begrüßt die Verhandlungen zu dem Anti-Counterfeiting Trade Agreement (ACTA), um einen sicheren und effizien- 
ten internationalen Rechtsrahmen zur Bekämpfung der Produktpiraterie zu schaffen. Dabei ist ihr eine substantielle Verbesserung des Schutzes geistigen Eigentums wichtiger als eine vorschnelle Verabschiedung.

Der Verhandlungsprozess wird so transparent wie möglich gestaltet. Die Europäische Kommission hat am 23. Juni 2008 eine Anhörung der beteiligten Kreise durchgeführt.

10. Abgeordnete

Grietje

Staffelt

(BÜNDNIS 90/

DIE GRÜNEN)
Welche Position bezieht die Bundesregierung zum sog. französischen Modell, das bei Urheberrechtsverletzungen im Internet ein abgestuftes Verfahren zur Durchsetzung geistiger Eigentumsrechte vorsieht, und plant die Bundesregierung Initiativen in dieser Hinsicht?

\section{Antwort des Parlamentarischen Staatssekretärs Alfred Hartenbach vom 15. September 2008}

Die Bundesregierung befürwortet die Entwicklung von Kooperationsverfahren zwischen Rechtsinhabern und Internetservicebetreibern zur Verfolgung und Bekämpfung von Urheberrechtsverletzungen im Internet. Bei dem in Frankreich entwickelten Modell, an dem auch die französische Regierung beteiligt ist, ist jedoch die Speicherung und Weitergabe personenbezogener Daten erforderlich. In Deutschland müssen entsprechende Kooperationsverfahren daher mit dem Fernmeldegeheimnis, dem Recht auf informationelle Selbstbestimmung und dem vom Bundesverfassungsgericht jüngst entwickelten Grundrecht auf Gewährleistung der Vertraulichkeit und Integrität informationstechnischer Systeme (sog. IT-Grundrecht) in Einklang gebracht werden. Der Gesetzgeber hat sich sowohl im Rahmen des Gesetzgebungsverfahrens zur Neuregelung der Telekommunikationsüberwachung und anderer verdeckter Ermittlungsmaßnahmen sowie zur Umsetzung der Richtlinie 2006/24/EG als auch im Rahmen des Gesetzgebungsverfahrens zur Verbesserung der Durchsetzung von Rechten geistigen Eigentums bewusst dagegen entschieden, die nach $\S 113 \mathrm{a}$ des Telekommunikationsgesetzes zu speichernden Daten (sog. Vorratsdaten) den Rechtsinhabern unmittelbar zugänglich zu machen. Die Bundesregierung erwägt zurzeit keine den Bestand dieser Entscheidung in Frage stellenden gesetzlichen Initiativen, weil es geboten erscheint, die Entscheidung des Bundesverfassungsgerichts über die dort anhängigen Verfassungsbeschwerden gegen das Gesetz zur Neuregelung der Telekommunikationsüberwachung und anderer verdeckter Ermittlungsmaßnahmen sowie zur Umsetzung der Richtlinie 2006/ 24/EG vom 21. Dezember 2007 abzuwarten.

11. Abgeordnete

\section{Grietje}

Staffelt

(BÜNDNIS 90/

DIE GRÜNEN)
Plant die Bundesregierung, in dieser Legislaturperiode eine weitere Reform des Urheberrechts, den sog. Dritten Korb, auf den Weg zu bringen, und wenn ja, welche Inhalte wird diese Reform haben? 


\section{Antwort des Parlamentarischen Staatssekretärs Alfred Hartenbach vom 15. September 2008}

Das Bundesministerium der Justiz wird noch im Herbst 2008 eine umfassende Konsultation mit den beteiligten Kreisen zu den EntschlieBungen des Deutschen Bundestages (Bundestagsdrucksache 16/5939) und des Bundesrates (Bundesratsdrucksache 582/07) einleiten. Nach Auswertung der Ergebnisse wird über das weitere Vorgehen zu entscheiden sein.

\section{Geschäftsbereich des Bundesministeriums der Finanzen}

12. Abgeordnete Cornelia

Behm

(BÜNDNIS 90/

DIE GRÜNEN)
Hält die Bundesregierung die Genehmigung der Mineralölsteuerermäßigung für die französische Landwirtschaft durch die EU-Kommission (laut AgraEurope vom 8. September 2008 zahlen französische Landwirte nur $66 \mathrm{Cent}$ pro Hektoliter Agrardiesel, der Unterglasgartenbau zahlt 11,9 Cent je MWh Erdgas und erhält für schweres Heizöl 16,65 Euro pro Tonne erstattet) im Vergleich zur Entscheidung der EU-Kommission zur deutschen Mineralölsteuerrückerstattung für Unterglasgartenbaubetriebe für gerechtfertigt, und wenn nein, wie gedenkt die Bundesregierung gegen diese Entscheidung vorzugehen?

\section{Antwort der Parlamentarischen Staatssekretärin Nicolette Kressl vom 15. September 2008}

Die Genehmigung der französischen Maßnahme durch die Europäische Kommission vom 16. Juli 2008 fußt beihilferechtlich auf der Rahmenregelung der Gemeinschaft für staatliche Beihilfen im Agrar- und Forstsektor für die Jahre 2007 bis 2013. Dort ist vorgesehen, dass ermäßigte Steuersätze auf als Kraft- oder Heizstoff für die landwirtschaftliche Primärerzeugung verwendete Erzeugnisse als mit Artikel 87 Abs. 3 Buchstabe c des EG-Vertrags vereinbar angesehen werden können, sofern die in der Energiesteuerrichtlinie (Richtlinie 2003/ 96/EG) festgelegten Mindeststeuerbeträge eingehalten werden und innerhalb des Landwirtschaftssektors keine Steuersatzdifferenzierung vorgenommen wird. Diese Grundsätze hat die Europäische Kommission für die Genehmigung der französischen Beihilferegelung ebenso wie bei der Genehmigung der deutschen Agrardieselregelung vom 30. April 2008 angewendet.

Bei der deutschen Mineralölsteuererstattung für Unternehmen der Land- und Forstwirtschaft zum Beheizen von Gewächshäusern oder geschlossenen Kulturräumen zur Pflanzenproduktion ( $\$ 58$ des Energiesteuergesetzes) ging es hingegen um eine sektorspezifische Begünstigung für Heizstoffe. 
13. Abgeordnete

Veronika

Bellmann

(CDU/CSU)
Wie sieht die Bundesregierung die nun von den Finanzministern der Länder geplante bundesweit einheitliche Besteuerung des Elterngeldes und die damit verbundene Abschaffung der sächsischen Sonderregelung, einen Sockelbetrag von 300 Euro nicht zu besteuern, und warum wird nicht diese Sonderregelung bundesweit eingeführt und unterstützt?

\section{Antwort der Parlamentarischen Staatssekretärin Nicolette Kressl vom 19. September 2008}

Elterngeld ist nach $\S 3$ Nr. 67 des Einkommensteuergesetzes (EStG) in voller Höhe steuerfrei. Es wird aber - wie auch andere Lohn- oder Einkommensersatzleistungen - dem Progressionsvorbehalt unterworfen (§ 32b Abs. 1 Buchstabe j EStG), da diese steuerfreie Leistung die wirtschaftliche Leistungsfähigkeit der berechtigten Personen erhöht. Progressionsvorbehalt bedeutet, dass das Elterngeld selbst nach wie vor steuerfrei bleibt, aber für das zu versteuernde Einkommen ein besonderer Steuersatz berechnet wird. Damit wird erreicht, dass der Grundsatz der Besteuerung nach der wirtschaftlichen Leistungsfähigkeit, der in der progressiven Gestaltung des Steuertarifs zum Ausdruck kommt, nicht durch die Steuerfreiheit bestimmter Bezüge durchbrochen wird. Mit dem Steuersatz soll die gesamte wirtschaftliche Leistungsfähigkeit erfasst werden, und zwar auch insoweit, als sie auf Bezügen beruht, die die steuerliche Bemessungsgrundlage nicht erhöhen.

Nach dem eindeutigen Gesetzeswortlaut wird das Elterngeld vollständig in den Progressionsvorbehalt einbezogen. Die sächsische Sonderregelung im Verwaltungswege widersprach dieser Gesetzeslage und konnte deshalb nicht aufrechterhalten werden. Für eine andere Handhabung wäre eine Gesetzesänderung nötig. Die Bundesregierung hält die bestehende Gesetzeslage aus Gründen der oben erläuterten steuerlichen Systematik des Progressionsvorbehalts für sachgerecht und sieht keinen Anlass für eine Änderung.

Insbesondere hält es die Bundesregierung sachlich nicht für geboten, den Elterngeldmindestbetrag von 300 Euro vom Progressionsvorbehalt auszunehmen. In den Fällen, in denen lediglich dieser Mindestbetrag an Eltern gezahlt wird, weil vor Geburt des Kindes kein Einkommen vorhanden war, handelt es sich zwar nicht um eine Einkommensersatzleistung. Das Mindestelterngeld ist aber, anders als beispielsweise das Erziehungsgeld, keine bedarfsabhängige Leistung. Vielmehr kommt es auch Familien zu, die wirtschaftlich sehr leistungsfähig sind. Familien mit geringem Einkommen sind hingegen vom Progressionsvorbehalt mangels steuerlicher Leistungsfähigkeit häufig gar nicht oder nur geringfügig belastet.

14. Abgeordneter

Hans-Michael

Goldmann

(FDP)
Hält die Bundesregierung an der Anweisung des Bundesministeriums der Finanzen zur steuerlichen Behandlung der Zahlungsansprüche fest, so dass für das zu Ende gegangene Wirtschaftsjahr 2007/2008 zwei Betriebsprämien versteuert werden müssten, obwohl die 
Betriebe nur eine Betriebsprämie erhalten haben und somit Belastungen für die Landwirtschaft in Höhe von bis zu 700 Mio. Euro entstehen?

\section{Antwort der Parlamentarischen Staatssekretärin Nicolette Kressl vom 19. September 2008}

Die obersten Finanzbehörden der Länder hatten zunächst einstimmig die Auffassung vertreten, dass aufgrund der Änderungen der rechtlichen Voraussetzungen für die Gewährung der Betriebsprämie im Zuge des GAP-Reformprozesses die Betriebsprämie künftig bereits zum Bilanzierungsstichtag 30. Juni 2008 zu berücksichtigen sei.

Diese Rechtsauffassung hätte bei bilanzierenden Landwirten im Wirtschaftsjahr 2007/2008 einmalig zur Erfassung von mehr als einer Betriebsprämie geführt. Nach einer groben Schätzung des Bundesministeriums der Finanzen hätten die daraus resultierenden Zinseffekte für die betroffenen Landwirte zu einer zusätzlichen wirtschaftlichen Belastung von insgesamt ca. 100 Mio. Euro geführt.

Die Problematik wurde deshalb am 12. September 2008 auf der Sitzung der Einkommensteuerreferatsleiter der obersten Finanzbehörden des Bundes und der Länder nochmals umfassend erörtert. Dabei wurde das Bundesministerium für Ernährung, Landwirtschaft und Verbraucherschutz eingehend zu den agrarrechtlichen Voraussetzungen für die Gewährung der Betriebsprämie befragt.

Aufgrund der daraus resultierenden zusätzlichen Erkenntnisse haben die Vertreter der obersten Finanzbehörden der Länder mehrheitlich entschieden, dass die Voraussetzungen für die Aktivierung der Betriebsprämie regelmäßig auch weiterhin erst mit Ablauf des Kalenderjahres erfüllt sind. Damit wird das einmalige Zusammenfallen von zwei Betriebsprämien in dem Gewinnermittlungszeitraum 2007/2008 vermieden.

Das Schreiben des Bundesministeriums der Finanzen vom 25. Juni 2008 wird entsprechend geändert.

15. Abgeordnete Christine

Scheel (BÜNDNIS 90/ DIE GRÜNEN)
Aus welchen steuerlichen Maßnahmen mit welchen zugehörigen Steuerausfällen setzt sich die in der Veröffentlichung des Bundesministeriums für Umwelt, Naturschutz und Reaktorsicherheit „Ökologische Industriepolitik - nachhaltige Politik für Innovation, Wachstum und Beschäftigung" auf S. 13 genannte Energiesteuerbefreiung beim Flugbenzin von knapp 7 Mrd. Euro zusammen?

\section{Antwort der Parlamentarischen Staatssekretärin Nicolette Kressl vom 12. September 2008}

Die Energiesteuerbefreiung für Energieerzeugnisse (Kerosin und Flugbenzin), die im Luftverkehr verwendet werden, beruht auf den 
Vorschriften des $\S 27$ Abs. 2 und des $\S 52$ des Gesetzes zur Neuregelung der Besteuerung von Energieerzeugnissen und zur Änderung des Stromsteuergesetzes (EnergieStG), wonach Energieerzeugnisse steuerfrei für die Luftfahrt, ausgenommen die private nicht gewerbliche Luftfahrt, verwendet werden dürfen.

Die Steuerbefreiung ist zudem Gegenstand einer Vielzahl von Luftverkehrsabkommen. Auf Gemeinschaftsebene ist sie in Artikel 14 Abs. 1 der Energiesteuerrichtlinie (RL 2003/96/EG) geregelt; danach dürfen internationale und innergemeinschaftliche Flüge nicht mit einer Energiesteuer belegt werden.

Artikel 14 Abs. 2 der Energiesteuerrichtlinie stellt den Mitgliedstaaten seit dem 1. Januar 2004 frei, reine Inlandsflüge der Kerosinsteuer zu unterwerfen. Dasselbe gilt für innergemeinschaftliche Flüge, wenn die betroffenen Mitgliedstaaten entsprechende bilaterale Verträge miteinander geschlossen haben. Bisher wurden solche bilateralen Verträge noch nicht geschlossen.

Im Subventionsbericht der Bundesregierung wird daher nur die Energiesteuerbefreiung des innerdeutschen Luftverkehrs als Subventionstatbestand genannt mit einem Subventionsvolumen von rd. 400 Mio. Euro.

16. Abgeordnete

Auf welchen Schätzgrundlagen beruht dies?

Christine

Scheel

(BÜNDNIS 90/

DIE GRÜNEN)

\section{Antwort der Parlamentarischen Staatssekretärin Nicolette Kressl vom 12. September 2008}

Die Angaben des Bundesministeriums für Umwelt, Naturschutz und Reaktorsicherheit beruhen auf Zahlen des Bundesamtes für Wirtschaft und Ausfuhrkontrolle, das auf der Basis des Mineralöldatengesetzes monatlich bei den in der Bundesrepublik Deutschland auf dem Mineralölmarkt tätigen Unternehmen Daten zu ihrer Geschäftstätigkeit erhebt. Danach wurden in 2006 rd. 8,5 Mio. Tonnen schwerer Flugturbinenkraftstoff (Kerosin) im Inland geliefert. Bei einer angenommenen Dichte des Kerosins von 0,8 und dem Regelsteuersatz für mittelschwere Öle von 654,50 Euro/1 000 Liter (§ 2 Abs. 1 Nr. 3 des Gesetzes zur Neuregelung der Besteuerung von Energieerzeugnissen und zur Änderung des Stromsteuergesetzes) ergibt sich ein Steuervolumen von rd. 7 Mrd. Euro.

17. Abgeordneter

Dr. Axel

Troost

(DIE LINKE.)
Mit welchen wirtschaftlichen und politischen Zielen bietet die Bundesrepublik Deutschland über die Finanzagentur seit dem 1. Juli 2008 die Tagesanleihe als neues Finanzprodukt für Privatkunden an, bzw. mit welchen wirtschaftlichen und politischen Zielen engagiert sich die Finanzagentur verstärkt im Privatkundengeschäft? 


\section{Antwort der Parlamentarischen Staatssekretärin Nicolette Kressl vom 19. September 2008}

Der Bund ist bereits seit dem Jahr 1969, in dem der erste Bundesschatzbrief emittiert wurde, im Privatkundengeschäft aktiv. Das Privatkundengeschäft trägt zur kostengünstigen Kreditaufnahme des Bundes bei, weil die Konditionen im Privatkundengeschäft regelmäßig günstiger für den Bund sind als diejenigen, die in der Kreditaufnahme an den internationalen Kapitalmärkten erzielbar sind. Allerdings macht das Privatkundengeschäft derzeit nur weniger als 2 Prozent der Bruttokreditaufnahme des Bundes aus, nachdem dieser Wert zu Beginn der 90er-Jahre noch deutlich höher gelegen hatte. Der aktuell geringe Wert ist im Wesentlichen wohl darauf zurückzuführen, dass es seit nunmehr gut 30 Jahren keine Aktualisierung der Privatkundenprodukte des Bundes gegeben hat. Die Tagesanleihe des Bundes ist ein neues Instrument, welches das Privatkundengeschäft wiederbeleben soll.

Die mit dem Bundesschuldenwesenmodernisierungsgesetz vom 12. Juli 2006 verbundene Intention ergibt sich im Übrigen aus der Gesetzesbegründung:

Das Privatkundengeschäft solle „unter wirtschaftlichen Bedingungen als wichtiges Element der Kreditaufnahme des Bundes künftig gestärkt werden, um eine Diversifizierung der Kreditaufnahme zu gewährleisten und dem Bürger zugleich eine sichere Geldanlage beim Bund anzubieten."

18. Abgeordneter Dr. Axel

Troost (DIE LINKE.)
Sieht die Bundesregierung eine Gefahr darin, dass die Tagesanleihe Genossenschaftsbanken und Sparkassen Einlagen entzieht und deren Refinanzierung erschwert (bitte begründet, warum ja oder nein) - so durch den doppelten Vorteil von täglicher Verfügbarkeit und täglichem Zinseszinseffekt der Tagesanleihe sowie der Bonität des Bundes, und wenn ja, welche Gegenmaßnahmen schlägt sie vor?

\section{Antwort der Parlamentarischen Staatssekretärin Nicolette Kressl vom 19. September 2008}

Die Tagesanleihe des Bundes gefährdet die Refinanzierung der Genossenschaftsbanken und Sparkassen nicht, zumal ihre Verzinsung eher im unteren Mittelfeld vergleichbarer kundenfreundlicher Angebote der Kreditinstitute an private Anleger liegt. Sie ist insbesondere nicht geeignet, in das Kernsegment insbesondere der Volks- und Raiffeisenbanken oder Sparkassen zu expandieren.

Die Kreditaufnahme des Bundes wird auch zukünftig zum weit überwiegenden Teil im Wege der Begebung von Bundeswertpapieren durch Auktionen erfolgen, die sich ausschließlich an Kreditinstitute und nicht an Privatkunden richten. 
19. Abgeordneter

Dr. Volker

Wissing

(FDP)
Wie oft wurden die gesetzlichen Regelungen zur Besteuerung von betrieblich genutzten Personenkraftwagen seit Beginn der 14. Legislaturperiode geändert, und um welche Änderungen hat es sich dabei jeweils gehandelt?

\section{Antwort der Parlamentarischen Staatssekretärin Nicolette Kressl vom 12. September 2008}

Seit Beginn der 14. Legislaturperiode wurden folgende Änderungen vorgenommen:

I. Einkommensteuerliche Änderungen

1. Gesetz zur Einführung einer Entfernungspauschale

Nach dem Gesetz zur Einführung einer Entfernungspauschale vom 21. Dezember 2000 (BGB1. I S. 1918) und dem Haushaltsbegleitgesetz 2004 vom 29. Dezember 2003 (BGB1. I S. 3076) sind ab dem Veranlagungszeitraum 2001 alle Aufwendungen für Fahrten zwischen Wohnung und Betriebsstätte durch die verkehrsmittelunabhängige Entfernungspauschale abgegolten.

2. Gesetz zur Eindämmung missbräuchlicher Steuergestaltungen

Durch das Gesetz zur Eindämmung missbräuchlicher Steuergestaltungen vom 28. April 2006 (BGB1. I S. 1095) wurde die Anwendung der sog. Listenpreisregelung beschränkt für Kraftfahrzeuge, die zu mehr als 50 vom Hundert betrieblich genutzt werden. Diese Regelung verhindert Steuergestaltungen, die nach der Änderung der Rechtsprechung des Bundesfinanzhofs (Urteil vom 2. Oktober 2003 IV R 13/03, BStB1 II 2003 S. 985) zur Zulässigkeit der Bildung gewillkürten Betriebsvermögens bei der Gewinnermittlung durch Einnahmeüberschussrechnung ( $\$ 4$ Abs. 3 EStG) möglich gewesen wären.

3. Steueränderungsgesetz 2007

Durch das Steueränderungsgesetz 2007 vom 19. Juli 2006 (BGBl. I S. 1652) wurde mit Wirkung vom 1. Januar 2007 die Entfernungspauschale abgeschafft. Für Fernpendler gilt eine Härtefallregelung. Danach können Aufwendungen für mehr als 20 Entfernungskilometer wie Betriebsausgaben abgezogen werden.

II. Umsatzsteuerliche Änderungen

Durch das Steuerentlastungsgesetz 1999/2000/2002 vom 24. März 1999 (BGBl. I S. 401) wurde mit $\S 15$ Abs. 1b des Umsatzsteuergesetzes (UStG) eine Beschränkung des Vorsteuerabzugs aus der Anschaffung und dem Betrieb von Fahrzeugen, die sowohl unternehmerisch als auch nichtunternehmerisch verwendet werden, eingeführt; im Gegenzug entfiel die Versteuerung der privaten Verwendung als unentgeltliche Wertabgabe nach $\S 3$ Abs. 9a UStG. Diese Maßnahme wurde durch das Steueränderungsgesetz 2003 vom 15. Dezember 2003 (BGBl. I S. 2645) mit Wirkung vom 31. Dezember 2003 aufgehoben. 


\section{Geschäftsbereich des Bundesministeriums für Wirtschaft und Technologie}

20. Abgeordnete Cornelia

Behm (BÜNDNIS 90/

DIE GRÜNEN)
Liegen der Bundesregierung in Anbetracht des massiven Preisanstiegs bei Düngemitteln um bis zu 100 bis 200 Prozent innerhalb der letzten Jahre Hinweise auf Preisabsprachen der Produzenten vor, und wenn ja, welche Handlungsmöglichkeiten dagegen vorzugehen sieht sie bzw. hat sie ergriffen?

\section{Antwort des Parlamentarischen Staatssekretärs Hartmut Schauerte vom 16. September 2008}

Der Bundesregierung liegen keine Hinweise auf Preisabsprachen der Produzenten von Düngemitteln vor. Preisabsprachen zwischen Herstellern von Düngemitteln würden einen Verstoß gegen das Kartellverbot - $\S 1$ des Gesetzes gegen Wettbewerbsbeschränkungen - darstellen. Für die Verfolgung und Ahndung eines solchen Verstoßes wäre das Bundeskartellamt zuständig. Das Bundeskartellamt führt derzeit kein Verfahren wegen des Verdachts auf Preisabsprachen durch.

21. Abgeordnete Ulrike Höfken (BÜNDNIS 90/ DIE GRÜNEN)
Was waren die Inhalte und die Ergebnisse des Besuchs der Delegation des Bundesministeriums für Wirtschaft und Technologie am 8. Juli 2008 im französischen Bure?

\section{Antwort des Staatssekretärs Jochen Homann vom 16. September 2008}

Deutsche Forschungseinrichtungen sind im Rahmen wissenschaftlichtechnischer Zusammenarbeit an Projekten im Untertagelabor Bure tätig. Sie führen seit 2001 eigene und gemeinsame Projekte mit anderen vor Ort tätigen internationalen Forschungseinrichtungen durch.

Am 8./9. Juli 2008 fand eine Besichtigung der Anlage und eine Vortrags- sowie Diskussionsveranstaltung zum Fortschritt der gemeinsamen FuE-Arbeiten im Untertagelabor Bure statt.

22. Abgeordnete

Ulrike

Höfken

(BÜNDNIS 90/

DIE GRÜNEN)
Welche Informationen hat die Bundesregierung über den Stand der Planungen und das konkrete Vorgehen von französischer Seite für ein Atommüllendlager in Bure, und gibt es Überlegungen seitens der Bundesregierung, in Zukunft deutschen Atommüll in einem möglichen Endlager in Lothringen zu lagern? 


\section{Antwort des Staatssekretärs Jochen Homann vom 16. September 2008}

1998 wurde durch Beschluss der französischen Regierung der Standort Bure (Departments Meuse/Haute-Marne) ausgewählt und 1999 mit dem Bau des Untertagelabors begonnen. Entsprechend der Betriebsgenehmigung sind ausschließlich wissenschaftlich-technische Arbeiten zulässig. Es handelt sich hierbei nicht um ein Versuchsendlager. Die Schächte sind im Durchmesser so geplant und ausgeführt worden, dass eine Verbringung von Behältern für hochradioaktive Abfälle nicht möglich ist.

Falls das untersuchte Tongestein sich als Endlagerwirtsgestein geeignet und genehmigungsfähig erweisen sollte, ist nach bisherigen Vorstellungen der französischen Regierung die Errichtung eines Endlagers in der Region Meuse/Haute-Marne geplant.

Die Bundesregierung ist der Auffassung, dass die in Deutschland anfallenden radioaktiven Abfälle im eigenen Land endzulagern sind.

23. Abgeordnete

Ulla

Jelpke

(DIE LINKE.)
Beabsichtigt die Bundesregierung, da ihr bislang keine Erkenntnisse darüber vorliegen, wie Sturmgewehre des Typs G 36 des Heckler \& Koch-Konzerns in den Besitz des georgischen Militärs geraten sind (lt. Antwort der Bundesregierung auf die schriftliche Frage 39 des Abgeordneten Hans-Christian Ströbele auf Bundestagsdrucksache 16/10199), sich mit der georgischen Regierung in Verbindung zu setzen, um anhand des schräg oberhalb des Magazins angebrachten Produzentenkürzels, der Produktionsnummer, des Herstellungsjahrs bzw. entsprechender Codebuchstaben Rückschlüsse auf den Hersteller zu gewinnen, bei dem anschließend über einen Einblick in die Exportbücher ermittelt werden könnte, wer Erstempfänger der Waffen war und auf welchem Weg sie nach Georgien gerieten (vgl. Interview mit dem Kleinwaffenexperten Jürgen Grässlin in junge Welt, 22. August 2008), oder hat sie bereits solche Schritte unternommen, und wenn ja, mit welchen Erkenntnissen?

\section{Antwort des Staatssekretärs Dr. Walther Otremba vom 16. September 2008}

Die Medienberichte zu G-36-Sturmgewehren bei Einheiten der georgischen Streitkräfte müssen sorgfältig untersucht und bewertet werden. Die zuständigen Stellen sind derzeit damit befasst. Die Bundesregierung hat keine Genehmigung zur Ausfuhr von Kleinwaffen nach Georgien erteilt. Die georgische Regierung wurde von der Bundesregierung in dieser Frage bislang nicht kontaktiert. 
24. Abgeordnete

Ute

Koczy

(BÜNDNIS 90/

DIE GRÜNEN)
Wie ist der aktuelle Stand bei der Umsetzung des Ilisu-Staudammprojekts, und wie sehen die Verfahrensschritte im Falle einer Kündigung der Verträge über die Hermesbürgschaft für das Ilisu-Staudammprojekt im Einzelnen aus?

\section{Antwort des Staatssekretärs Dr. Walther Otremba vom 17. September 2008}

Die Exportkreditversicherungen (ECAs) aus Österreich, der Schweiz und Deutschland haben seit Beginn des Ilisu-Staudammprojektes strenge Anforderungen an die Übernahme einer Exportkreditgarantie gestellt. Die Türkei hat sich zur Einhaltung dieser Standards den ECAs gegenüber verpflichtet.

Durch den Verzug bei der notwendigen Umsetzung von vorbereitenden Maßnahmen in den Bereichen Umsiedlung, Umwelt und Kulturgüter bei gleichzeitiger Koordinierung von Bauaktivitäten und der Einleitung von Enteignungen wurde von dem vereinbarten Verfahren abgewichen. Es ist daher notwendig geworden, die vorliegenden Bauzeitpläne anzupassen. Über die konkreten Korrekturmaßnahmen wird derzeit verhandelt.

Die Verträge sehen vor, dass bei nachhaltigen Verstößen gegen die getroffenen Vereinbarungen eine förmliche Umweltstörungsanzeige an das Lieferkonsortium versendet werden kann. Beseitigt der türkische Bauherr in der Folge die Störung nicht, ist eine Anweisung durch die ECAs zur Suspendierung der Lieferverträge möglich.

Zum aktuellen Zeitpunkt besteht noch die Möglichkeit, eine einvernehmliche Lösung zu finden.

25. Abgeordneter

Hans-Joachim

Otto

(Frankfurt)

(FDP)
Welche Pläne zum Rückbau der Hauptverteiler in der Bundesrepublik Deutschland durch die Deutsche Telekom AG sind der Bundesregierung bzw. der Bundesnetzagentur bekannt, und wie bewertet die Bundesregierung diese Pläne aus regulatorischer, wirtschafts- und wettbewerbspolitischer Sicht?

\section{Antwort des Parlamentarischen Staatssekretärs Hartmut Schauerte vom 18. September 2008}

Derzeit liegen weder der Bundesregierung noch der Bundesnetzagentur Informationen über konkrete Pläne zum Rückbau der Hauptverteiler durch die Deutsche Telekom AG vor. Allerdings soll es in der Sache wohl Gespräche zwischen der Deutschen Telekom AG und den Wettbewerberverbänden geben. Grundsätzlich bewertet die Bundesregierung die Pläne der Deutschen Telekom AG und ihrer Wettbewerber, in den Aufbau von Glasfasernetzen zu investieren, als überaus positiv. Dies wird zu einem Qualitätssprung in der Breitbandkommunikation führen und hat das Potential, einen Wachstumsschub für den Wirtschaftsstandort Deutschland zu entfalten. 
Die damit einhergehenden Veränderungen im Anschlussnetz, wozu auch der Rückbau von Hauptverteilern zählt, sind so zu gestalten, dass keine Wettbewerbsverzerrungen auftreten. So müssen etwa aus regulatorischer bzw. regulierungsrechtlicher Sicht Rück- bzw. Umbaupläne so rechtzeitig, umfassend und transparent angekündigt werden, dass sich die davon betroffenen Wettbewerber auf die Veränderungen einstellen können.

Eine detaillierte Bewertung ist erst in Kenntnis der genauen Pläne möglich und bleibt darüber hinaus den ggf. erforderlich werdenden konkreten Regulierungsverfahren vorbehalten.

26. Abgeordneter

Paul

Schäfer

(Köln)

(DIE LINKE.)
In welchen Ländern werden/wurden nach Kenntnis der Bundesregierung Gewehre vom Typ G36 (in seinen unterschiedlichen Versionen) hergestellt oder mithilfe zugelieferter Komponenten endmontiert?

\section{Antwort des Parlamentarischen Staatssekretärs \\ Hartmut Schauerte \\ vom 17. September 2008}

Bislang wurden Gewehre vom G36 außer in Deutschland nur in Spanien in Lizenz gefertigt oder endmontiert.

27. Abgeordneter

Paul

Schäfer

(Köln)

(DIE LINKE.)
Wann und durch wen erhielt die Bundesregierung erstmals Kenntnis von der Tatsache, dass georgische Sicherheitskräfte über Gewehre des Typs G36 verfügen?

\section{Antwort des Parlamentarischen Staatssekretärs \\ Hartmut Schauerte \\ vom 17. September 2008}

In der Woche vor der Ausstrahlung des ARD-Magazins „Report“ am 18. August 2008 erhielt die Bundesregierung durch den betreffenden Journalisten Anfragen und Fotos zu dem Sachverhalt.

28. Abgeordneter

Paul

Schäfer

(Köln)

(DIE LINKE.)
Welche Kenntnisse hat die Bundesregierung derzeit darüber, wie diese Gewehre nach Georgien gelangten, und ob dafür die nach deutscher Rechtslage erforderlichen Voraussetzungen erfüllt waren? 


\section{Antwort des Parlamentarischen Staatssekretärs \\ Hartmut Schauerte \\ vom 17. September 2008}

Die Bundesregierung hat keine Genehmigung zur Ausfuhr von Gewehren des Typs G36 nach Georgien erteilt. Auf welchem Wege die Waffen nach Georgien gelangt sind, entzieht sich bislang ihrer Kenntnis.

29. Abgeordneter

Hat die Bundesregierung Kenntnis von Serien-

Paul nummern der in Georgien genutzten Geweh-

Schäfer

(Köln) ren des Typs G36, und falls ja, wie lauten

(DIE LINKE.) diese?

\section{Antwort des Parlamentarischen Staatssekretärs \\ Hartmut Schauerte \\ vom 17. September 2008}

Die Bundesregierung hat darüber keine Kenntnis.

30. Abgeordneter

Hans-Christian

Ströbele

(BÜNDNIS 90/

DIE GRÜNEN)
Welche Schritte unternahm die Bundesregierung nebst nachgeordneter Stellen zur Aufklärung der Herkunft „deutscher“ G-36-Gewehre in Georgien (vgl. meine schriftliche Frage 39 auf Bundestagsdrucksache 16/10199), insbesondere etwaige dahingehende Auskunftsersuchen an die georgische Regierung auch bezüglich der Seriennummern dieser Gewehre, und welche Ergebnisse sowie Reaktionen erbrachte dies?

\section{Antwort des Parlamentarischen Staatssekretärs Hartmut Schauerte vom 18. September 2008}

Die Medienberichte zu G-36-Sturmgewehren bei Einheiten der georgischen Streitkräfte müssen sorgfältig überprüft und bewertet werden. Die Untersuchungen dazu sind noch nicht abgeschlossen. Soweit es die Bundesregierung für sinnvoll erachtet, wird sie in dieser Sache ggf. auch andere Staaten kontaktieren. 


\section{Geschäftsbereich des Bundesministeriums für Arbeit und Soziales}

31. Abgeordnete

Brigitte

Pothmer

(BÜNDNIS 90/

DIE GRÜNEN)
Wann ist mit dem Einvernehmen des Bundesministeriums der Finanzen für die Entsperrung von 1500 Stellen für Personal der Bundesagentur für Arbeit in den ARGEn und den Agenturen in getrennter Aufgabenwahrnehmung (AAgAw) zu rechnen, nachdem die dafür erforderlichen Auflagen des Haushaltsgebers von der Bundesagentur erfüllt worden sind (vgl. Ausschussdrucksache 16(11)1068 vom 22. August 2008)?

\section{Antwort des Parlamentarischen Staatssekretärs Klaus Brandner vom 16. September 2008}

Das Bundesministerium für Arbeit und Soziales hat im Einvernehmen mit dem Bundesministerium der Finanzen mit Schreiben vom 9. Juli 2008 an die Bundesagentur für Arbeit der Aufhebung der Sperre für die 1500 Stellen der zweiten Tranche zugestimmt.

32. Abgeordnete

Brigitte

Pothmer

(BÜNDNIS 90/

DIE GRÜNEN)
Welche einzelnen Tätigkeiten des operativen Geschäfts der ARGEn/AAgAw (Fallmanager, persönlicher Ansprechpartner, Arbeitsvermittler, Assistenzkraft, Teamleiter, Führungskräfte, Fahrer ...) bilden sich jeweils in welcher Größenordnung in der dem vorläufigen ganzheitlichen Orientierungsschlüssel zugrunde liegenden Mitarbeiterkapazitäten ab (vgl. Ausschussdrucksache 16(11)1068)?

\section{Antwort des Parlamentarischen Staatssekretärs Klaus Brandner vom 16. September 2008}

Der in der Ausschussdrucksache angeführte vorläufige ganzheitliche Betreuungsschlüssel von 1:79 bezieht sich nur auf das operative Personal in den Arbeitsgemeinschaften und Agenturen mit getrennter Aufgabenwahrnehmung. Hierzu zählen z. B. nicht Fahrer oder Controller. Zum operativen Personal gezählt werden nach einer Auswertung der Bundesagentur für Arbeit von Januar 2008 rd. 30500 Fachkräfte (darunter auch Teamleiter und andere Führungskräfte) und 22600 Assistenzkräfte.

33. Abgeordnete

Brigitte

Pothmer (BÜNDNIS 90/ DIE GRÜNEN)
Welches bundesdurchschnittliche Betreuungsverhältnis ergibt sich (auf Basis der Datengrundlage zu Tabelle 1 der Ausschussdrucksache 16(11) 1068, wenn alle erwerbsfähigen Hilfebedürftigen und alle Mitarbeiterkapazitäten im operativen Bereich analog zum vorläufigen 
ganzheitlichen Orientierungsschlüssel mit dem Referenzwert 1:79 in die Berechnung einbezogen werden?

\section{Antwort des Parlamentarischen Staatssekretärs Klaus Brandner vom 16. September 2008}

Auf Basis der Datengrundlage zu Tabelle 1 der Ausschussdrucksache 16(11)1068 kann der vorläufige ganzheitliche Betreuungsschlüssel nicht errechnet werden, weil dem Bundesministerium für Arbeit und Soziales für den Personalstand im Mai 2008 nicht die Zahl des gesamten operativen Personals in der unterstellten Abgrenzung vorliegt und diese in der kurzen Frist auch nicht ermittelt werden konnte. Das Bundesministerium für Arbeit und Soziales wird in Kürze im Einvernehmen mit dem Bundesministerium der Finanzen die bisherigen Betreuungsschlüssel für die Bereiche Vermittlung unter 25-Jähriger, Vermittlung über 25-Jähriger und Leistungsgewährung präzisieren und verbindlich vorgeben.

34. Abgeordnete

Brigitte

Pothmer

(BÜNDNIS 90/

DIE GRÜNEN)
Beabsichtigt die Bundesregierung die vom Haushaltsausschuss am 12. Dezember 2007 für das Haushaltsjahr 2008 vorgegebene Obergrenze für befristete Beschäftigungsverhältnisse für BA-Personal in den ARGEn/AAgAw von 11400 Stellen durch das Ziel eines Befristungsanteils von maximal 10 Prozent abzulösen (ca. 3500 befristete Stellen), und welcher Handlungsbedarf ergibt sich daraus in den Haushaltsberatungen für 2009, 2010 und 2011 für die Etatisierung von Dauerstellen?

\section{Antwort des Parlamentarischen Staatssekretärs Klaus Brandner vom 16. September 2008}

Die Bundesregierung beabsichtigt, in einem mittelfristigen Zeitraum das Personal in den ARGEn und AAGAw durch weitere Bereitstellung von festen Stellen zu stabilisieren. Der konkrete Handlungsbedarf für die kommenden Jahre wird derzeit auf der Grundlage aktualisierter Personaldaten bestimmt.

\section{Geschäftsbereich des Bundesministeriums der Verteidigung}

35. Abgeordneter

Reinhard

Grindel

(CDU/CSU)
Welche Schlüsse zieht die Bundesregierung aus dem Plakat „Schritt zur Abrüstung“ des Büros für antimilitaristische Maßnahmen (BamM) und des Landesverbandes BerlinBrandenburg der Deutschen Friedensgesellschaft - Vereinigte KriegsdienstgegnerInnen 
(DFG-VK) und der Distanzierung von diesem Plakat durch den Bundessprecherkreis der DFG-VK?

36. Abgeordneter

Reinhard

Grindel

$(\mathrm{CDU} / \mathrm{CSU})$
Plant die Bundesregierung angesichts des menschenverachtenden Materials des BamM und des Landesverbandes Berlin-Brandenburg der DFG-VK zur „Öffentlichkeitsarbeit“ rechtliche Schritte gegen die genannten Vereinigungen einzuleiten?

\section{Antwort des Parlamentarischen Staatssekretärs \\ Thomas Kossendey \\ vom 12. September 2008}

Ihre Empörung über das unzweifelhaft geschmacklose Plakat „Schritt zur Abrüstung" auf der gemeinsamen Internetseite des Büros für antimilitaristische Maßnahmen (BamM) und des Landesverbandes BerlinBrandenburg der Deutschen Friedensgesellschaft - Vereinigte KriegsgegnerInnen (DFG-VK) teile ich.

Das Plakat ist der Bundesregierung bereits seit längerem bekannt. Aufgrund der aktuellen Presseberichterstattung ist es nunmehr (erneut) in den Blickpunkt der Öffentlichkeit gelangt. Ein konkreter Bezug zu aktuellen Todesfällen bei der Bundeswehr wurde in der Vergangenheit und wird auch aktuell durch die Betreiber der Internetseite nicht hergestellt.

Das Plakat war seit dem Jahr 2003 bereits mehrfach Gegenstand strafrechtlicher Überprüfung im hiesigen Ressort. Im Ergebnis wurde von einer Strafanzeige seitens des Bundesministeriums der Verteidigung gegen die Betreiber der Internetseite abgesehen. Ausschlaggebend hierfür war die Rechtsprechung des Bundesverfassungsgerichts, die in vergleichbaren Fällen (Stichwort „Soldaten sind Mörder") dem Grundrecht auf freie Meinungsäußerung im Rahmen der öffentlichen und politischen Meinungsbildung einen weiten Raum zugesteht, so dass von einem strafrechtlich relevanten Verhalten nicht ausgegangen wurde. Durch eine hiesigerseits gestellte - und im Ergebnis mit hoher Wahrscheinlichkeit erfolglose - Strafanzeige hätten die Betreiber der Internetseite lediglich eine erhöhte Aufmerksamkeit und ein zusätzliches Forum für ihre Aktivitäten erlangt. Dies galt es auch im Interesse unserer Soldatinnen und Soldaten zu vermeiden.

Aus den vorgenannten Gründen beabsichtigt die Bundesregierung nicht, rechtliche Schritte einzuleiten.

37. Abgeordneter

Reinhard

Grindel

(CDU/CSU)
Hat die Bundesregierung Erkenntnisse über Finanzierungsquellen des BamM und/oder des Landesverbandes Berlin-Brandenburg der DFG-VK direkt oder indirekt aus Mitteln der öffentlichen Hand? 


\section{Antwort des Parlamentarischen Staatssekretärs \\ Thomas Kossendey \\ vom 12. September 2008}

Die Bundesregierung hat keine Erkenntnisse darüber, dass die BamM und/oder der Landesverband Berlin-Brandenburg der DFG-VK Zuschüsse oder Leistungen aus öffentlichen Mitteln des Bundes erhalten haben bzw. weiterhin erhalten.

38. Abgeordneter Henning

Otte (CDU/CSU)
Welchen Handlungsbedarf sieht die Bundesregierung auf Grund der Aktivitäten des Büros für antimilitaristische Maßnahmen (BamM) und des Landesverbands Berlin-Brandenburg der DFG-VK (Deutsche Friedensgesellschaft Vereinigte KriegsdienstgegnerInnen), insbesondere in Bezug auf das von der BamM herausgegebene Plakat zum Tod von Bundeswehrsoldaten?

Sieht die Bundesregierung bei den Aktivitäten der BamM und des DFG-VK mit seinen Landesverbänden einen Straftatbestand erfüllt, und wenn ja, wird sie entsprechende Strafanzeigen stellen?

\section{Antwort des Parlamentarischen Staatssekretärs Thomas Kossendey vom 12. September 2008}

Ihre Empörung über das unzweifelhaft geschmacklose Plakat „Schritt zur Abrüstung" auf der gemeinsamen Internetseite des Büros für antimilitaristische Maßnahmen (BamM) und des Landesverbandes BerlinBrandenburg der Deutschen Friedensgesellschaft - Vereinigte KriegsgegnerInnen (DFG-VK) teile ich.

Das Plakat ist der Bundesregierung bereits seit längerem bekannt. Aufgrund der aktuellen Presseberichterstattung ist es nunmehr (erneut) in den Blickpunkt der Öffentlichkeit gelangt. Ein konkreter Bezug zu aktuellen Todesfällen bei der Bundeswehr wurde in der Vergangenheit und wird auch aktuell durch die Betreiber der Internetseite nicht hergestellt.

Das Plakat war seit dem Jahr 2003 bereits mehrfach Gegenstand strafrechtlicher Überprüfung im hiesigen Ressort. Im Ergebnis wurde von einer Strafanzeige seitens des Bundesministeriums der Verteidigung gegen die Betreiber der Internetseite abgesehen. Ausschlaggebend hierfür war die Rechtsprechung des Bundesverfassungsgerichts, die in vergleichbaren Fällen (Stichwort „Soldaten sind Mörder") dem Grundrecht auf freie Meinungsäußerung im Rahmen der öffentlichen und politischen Meinungsbildung einen weiten Raum zugesteht, so dass von einem strafrechtlich relevanten Verhalten nicht ausgegangen wurde. Durch eine hiesigerseits gestellte - und im Ergebnis mit hoher 
Wahrscheinlichkeit erfolglose - Strafanzeige hätten die Betreiber der Internetseite lediglich eine erhöhte Aufmerksamkeit und ein zusätzliches Forum für ihre Aktivitäten erlangt. Dies galt es auch im Interesse unserer Soldatinnen und Soldaten zu vermeiden.

Aus den vorgenannten Gründen beabsichtigt die Bundesregierung nicht, rechtliche Schritte einzuleiten.

40. Abgeordneter

Henning

Otte

(CDU/CSU)
Erhalten das Büro für antimilitaristische Maßnahmen (BamM), die DFG-VK (Deutsche Friedensgesellschaft - Vereinigte KriegsdienstgegnerInnen) insbesondere der Landesverband Berlin-Brandenburg der DFG-VK öffentliche Gelder für ihre Arbeit, und wenn ja, soll diese öffentliche Unterstützung (bitte genauen Betrag angeben) eingestellt werden?

\section{Antwort des Parlamentarischen Staatssekretärs \\ Thomas Kossendey \\ vom 12. September 2008}

Die Bundesregierung hat keine Erkenntnisse darüber, dass die BamM und/oder der Landesverband Berlin-Brandenburg der DFG-VK Zuschüsse oder Leistungen aus öffentlichen Mitteln des Bundes erhalten haben bzw. weiterhin erhalten.

41. Abgeordneter Dr. Rainer Stinner (FDP)
Teilt die Bundesregierung die Ansicht des Parlamentarischen Staatssekretärs beim Bundesminister der Verteidigung, Thomas Kossendey, „Das Mandat für OEF am Horn von Afrika schließt den Kampf gegen Piraterie durch die Deutsche Marine ausdrücklich aus" (FAZ.net vom 25. Juni 2008), und wenn ja, auf welche konkrete Formulierung im Mandat stützt sie diese Ansicht?

\section{Antwort des Parlamentarischen Staatssekretärs Christian Schmidt vom 15. September 2008}

Das zuletzt am 12. Oktober 2007 verlängerte Mandat des Deutschen Bundestages für den Einsatz bewaffneter deutscher Streitkräfte im Rahmen der Operation Enduring Freedom (OEF) sieht nur die Bekämpfung des internationalen Terrorismus und nicht die Pirateriebekämpfung vor, so dass diese im Rahmen dieses Mandats nicht erfolgen kann.

42. Abgeordneter Dr. Rainer Stinner (FDP)
Teilt die Bundesregierung die Ansicht, die der Bundesminister der Verteidigung, Dr. Franz Josef Jung, in Kabul geäußert hat: „Hier sind wir in einer asymmetrischen Bedrohungslage und deshalb auch im Kampf gegen den Terro- 
rismus“ (heute-journal, 3. September 2008), und wenn ja, wie vereinbart dies die Bundesregierung mit ihrer bisherigen Darstellung, der Kampf gegen den Terrorismus sei nicht Teil des ISAF-Mandates, sondern erfolge nur unter OEF?

\section{Antwort des Parlamentarischen Staatssekretärs Thomas Kossendey vom 12. September 2008}

Das aktualisierte und am 9. September 2008 von der Bundesregierung beschlossene Afghanistankonzept nennt im Vorwort drei Ziele für das umfangreiche zivil-militärische Engagement Deutschlands in Afghanistan:

„Wir unterstützen Afghanistan dabei, die Lebensverhältnisse für die eigene Bevölkerung zu verbessern. Wir beteiligen uns an den Anstrengungen der internationalen Staatengemeinschaft, regionale Stabilität und Sicherheit in einem schwierigen Umfeld zu gewährleisten; und wir verteidigen unsere eigenen Sicherheitsinteressen, indem wir zur Eindämmung des weltweiten Terrorismus beitragen. Afghanistan darf nicht erneut zum Rückzugsraum des internationalen Tourismus werden!“

Die Bundesregierung hat damit klargestellt, dass das gesamte deutsche Engagement in und für Afghanistan einen Beitrag zum weltweiten Kampf gegen den Terrorismus leistet. Der militärische Beitrag der deutschen ISAF-Kräfte besteht dabei allerdings nicht in einer aktiven Bekämpfung von Terroristen, sondern in der Unterstützung der afghanischen Regierung bei der Schaffung und Erhaltung eines sicheren Umfelds, in dem Wiederaufbau und Entwicklung möglich sind. Unbenommen davon gilt für die ISAF-Kräfte das Recht zur Selbstverteidigung, dass die Abwehr einer terroristischen Bedrohung selbstverständlich einschließt.

\section{Geschäftsbereich des Bundesministeriums für Familie, Senioren, Frauen und Jugend}

43. Abgeordnete

Britta

Haßelmann

(BÜNDNIS 90/

DIE GRÜNEN)
Distanziert sich die Bundesregierung von der öffentlichen Einlassung des Staatssekretärs im Bundesministerium für Familie, Senioren, Frauen und Jugend, Gerd Hoofe, „Die direkte, dauerhafte und abhängige Förderung oder Zuwendung kann nur die absolute Ausnahme sein, da sich sonst zu starke Abhängigkeiten entwickeln, die zur Erosion der Zivilgesellschaft führen" (Protokoll der 30. Sitzung des „Arbeitskreises Bürgergesellschaft und aktivierender Staat" der Friedrich Ebert Stiftung am 6. Juni 2008 zur institutionellen und infra- 
strukturellen Förderung des bürgerschaftlichen Engagements), und falls nein, welche Kriterien müssen nach Auffassung der Bundesregierung vorliegen, damit die absolute Ausnahme einer direkten, dauerhaften und abhängigen Förderung oder Zuwendung gegeben ist?

\section{Antwort des Parlamentarischen Staatssekretärs \\ Dr. Hermann Kues \\ vom 16. September 2008}

Die Bundesregierung distanziert sich von dieser Äußerung, die die geltende Rechtslage wiedergibt, nicht. Nach dem im Zuwendungsrecht geltenden Subsidiaritätsprinzip in Verbindung mit dem Wirtschaftlichkeitsgrundsatz dürfen Zuwendungen nur nachrangig und nur so lange und so weit gewährt werden, als dass sie zur Erfüllung der Aufgaben notwendig und angemessen sind.

Dabei sind institutionelle Dauerförderungen ausgeschlossen, sofern sie nicht ausdrücklich im Haushaltsplan ausgewiesen sind. Projektförderungen hingegen sind immer nur auf einen bestimmten Zeitraum ausgerichtet und zulässig.

44. Abgeordnete

Britta

Haßelmann

(BÜNDNIS 90/

DIE GRÜNEN)
In welcher Höhe und nach welchen Kriterien werden Organisationen und Vereine im Einzelnen - neben den im Kapitel 1702 des Bundeshaushalts 2009 genannten elf institutionell geförderten Zuwendungsempfängern - institutionelle Förderung oder Projektförderung erhalten?

\section{Antwort des Parlamentarischen Staatssekretärs \\ Dr. Hermann Kues \\ vom 16. September 2008}

Der Kabinettsentwurf des Haushalts 2009 sieht im Einzelplan 17 bei Kapitel 1702 Ausgaben in Höhe von 382826 Euro vor. Hiervon entfallen auf:

- 11 institutionelle Zuwendungsempfänger:

20597 Euro

- Projektförderung:

284375 Euro

- sonstiges (gesetzliche Leistungen, Jugendwerke): 77854 Euro.

Die Kriterien der Projektförderung sind in Förderrichtlinien zu den einzelnen Politikbereichen (Jugend, Familie, Senioren, Gleichstellung) des Bundesministeriums für Familie, Senioren, Frauen und Jugend (BMFSFJ) niedergelegt.

Darüber hinaus gibt es noch für einzelne Programme gesonderte Richtlinien (z. B. Mehrgenerationenhäuser). 
45. Abgeordnete

Britta

Haßelmann

(BÜNDNIS 90/

DIE GRÜNEN)
Warum und nach welchen Kriterien wurden bei einigen Organisationen die Projektförderung im Kapitel 1702 des Bundeshaushalts 2009 nicht fortgesetzt, obwohl deren Förderung eigentlich für einen längeren Zeitraum vorgesehen war, wie beispielsweise für die „Nationale Kontakt- und Informationsstelle zur Anregung und Unterstützung von Selbsthilfegruppen“ (NAKOS), deren Förderung eigentlich bis 2010 laufen sollte?

\section{Antwort des Parlamentarischen Staatssekretärs \\ Dr. Hermann Kues \\ vom 16. September 2008}

Grundsätzlich werden Projektförderungen entsprechend den Vorgaben der Bundeshaushaltsordnung nur zeitlich begrenzt ausgesprochen und durchgeführt. Ein Anspruch auf Weiterförderung ist nicht gegeben, da sonst die Gefahr bestünde, dass mit der Förderung einer Dauerförderung etabliert würde. Eine vorzeitige Beendigung kann in Betracht kommen, sofern aus dem Projektverlauf ersichtlich wird, dass der Zuwendungszweck (Förderziel) nicht erreichbar ist und daher ein erhebliches Bundesinteresse nicht mehr besteht.

Für das Projekt NAKOS wurde ursprünglich eine Förderung bis zum 31. Dezember 2009 beantragt. Der Zuwendungsbescheid ist zunächst jedoch nur bis 31. Dezember 2008 terminiert worden. Das nachhaltige Aufgabenverständnis von NAKOS, die Zusammenarbeit von Selbsthilfegruppen, Selbsthilfekontaktstellen und Versorgungs- und Beratungseinrichtungen auf örtlicher Ebene zu fördern, besteht unabhängig von der Projektkonstruktion und ist nicht nur befristet auf den Projektzeitraum zu verkürzen. NAKOS hat in Gesprächen selbst eingeräumt, dass die laufende Förderung wesentlich dem Erhalt der eigenen Personalstruktur dient und damit die Erwartung entstehen kann, auf Dauer in dieser Form gefördert zu werden. Eine Reihe der Aufgaben werden nach Aussage von NAKOS in ähnlicher Form auch von anderen Organisationen, wie dem Paritätische Wohlfahrtsverband, der vom BMFSFJ aus dem Bereich der Zentralstellenförderung unterstützt wird, erfolgreich wahrgenommen.

Mit der Entscheidung über die Beendigung der Förderung wird keine negative Aussage über die Qualität der Arbeit von NAKOS getroffen. NAKOS ist aber ganz wesentlich im Gesundheitsbereich tätig und sollte Förderoptionen aus dem Gesundheitsbereich nutzen. Die Reformen der Gesundheitspolitik, insbesondere die Einrichtung von Pflegestützpunkten, ermöglichen auch in Zukunft eine stärkere Förderung der Selbsthilfe. 


\section{Geschäftsbereich des Bundesministeriums für Verkehr, Bau und Stadtentwicklung}

46. Abgeordneter

Lutz

Heilmann

(DIE LINKE.)

\begin{abstract}
Welche Ergebnisse hatten die Untersuchungen der Bundesregierung über den möglichen Entfall von ein oder zwei Schleusenstandorten am Elbe-Lübeck-Kanal (s. Antwort der Bundesregierung vom 11. September 2006 auf meine schriftliche Frage 70 auf Bundestagsdrucksache 16/2585), und welche Anpassungen des Kanalbetts sind bei der von der Bundesregierung präferierten Variante zum Ersatz der Schleusen erforderlich?
\end{abstract}

\section{Antwort der Parlamentarischen Staatssekretärin Karin Roth vom 11. September 2008}

Die Schleusen im Nordabstieg des Elbe-Lübeck-Kanals (Donnerschleuse, Schleuse Behlendorf, Schleuse Berkenthin, Schleuse Krummesse und Schleuse Büssau) wurden instand gesetzt und haben noch eine Restnutzungsdauer von rund 20 Jahren. Ob und welche Standorte bei einem Einsatz dieser Schleusen ggf. entfallen können und welche Anpassungsmaßnahmen am Kanalbett dies zur Folge haben würde, wird noch untersucht.

47. Abgeordneter Ingbert Liebing (CDU/CSU)
Wann hat die Bundesregierung festgestellt, dass die am 12. August 2008 begonnene Aktion von Greenpeace, Steine im Flora-FaunaHabitat-Gebiet „Sylter Außenriff“ in der Ausschließlichen Wirtschaftszone zu versenken, illegal ist?

\section{Antwort der Parlamentarischen Staatssekretärin Karin Roth vom 12. September 2008}

Das Bundesamt für Seeschifffahrt und Hydrographie (BSH) wurde am Mittag des 12. August 2008 von der Bundespolizei über die Greenpeace-Aktion unterrichtet. Das BSH hat noch am selben Tag festgestellt, dass die Aktion gegen $\S 4$ des Hohe-See-Einbringungsgesetzes verstößt.

48. Abgeordneter Ingbert

Liebing $(\mathrm{CDU} / \mathrm{CSU})$
Welche Schritte hat die Bundesregierung zu welchem Zeitpunkt unternommen, um in einem einheitlichen geschlossenen Handeln der Verwaltungs- und Vollzugsorgane die nach Auffassung der Bundesregierung illegale Aktion zu stoppen? 


\section{Antwort der Parlamentarischen Staatssekretärin Karin Roth vom 12. September 2008}

Das BSH hat noch am 12. August 2008 in einem ersten Schritt Greenpeace auf den Rechtsverstoß hingewiesen. Nach Prüfung und Klärung der Rechtsgrundlage für eine vollziehbare Untersagungsverfügung hat in einem weiteren Schritt die Bundesregierung den nach Maßgabe des Seerechtsübereinkommens der Vereinten Nationen in erster Linie zuständigen Flaggenstaat des für den Transport und die Einbringung der Steine genutzten Schiffes aufgefordert, die gegen das Internationale Übereinkommen vom 29. Dezember 1972 über die Verhütung der Meeresverschmutzung durch das Einbringen von Abfällen und anderen Stoffen verstoßenden Handlungen zu unterbinden. Außerdem hat die Wasser- und Schifffahrtsdirektion Nord auf der Grundlage des allgemeinen Gefahrenabwehrtatbestandes des Seeaufgabengesetzes für die deutsche Ausschließliche Wirtschaftszone kurzfristig geeignete Maßnahmen zur Durchsetzung des Einbringungsverbots getroffen und den Verantwortlichen für den Gesetzesverstoß am Freitag, den 5. September 2008 eine sofort vollziehbare Untersagungsverfügung zugestellt.

49. Abgeordneter

Horst

Meierhofer

(FDP)
Gedenkt die Bundesregierung die nicht mehr nutzbare Donau-Wehrbrücke (Protzenweiher Brücke) wieder aufzubauen, und wenn ja, wie lautet der genaue Zeitplan?

\section{Antwort der Parlamentarischen Staatssekretärin Karin Roth vom 15. September 2008}

Ja. Zurzeit laufen die notwendigen Vorbereitungen und Abstimmungsgespräche mit der Stadt Regensburg. Deswegen können zum jetzigen Zeitpunkt noch keine Aussagen zu einem genauen Zeitplan gemacht werden.

\section{Geschäftsbereich des Bundesministeriums für Umwelt, Naturschutz und Reaktorsicherheit}

50. Abgeordnete

Angelika

Brunkhorst

(FDP)
Soll nach Auffassung der Bundesregierung nach der Einigung zwischen dem Bundesministerium für Bildung und Forschung (BMBF) und dem Bundesministerium für Umwelt, Naturschutz und Reaktorsicherheit (BMU) auf einen Betreiberwechsel für die Asse (vgl. BMU-Pressdienst Nr. 185/08 vom 4. September 2008) die Übertragung der finanziellen Verantwortung vom BMBF auf das BMU bereits in den aktuellen Beratungen über den Bundeshaushalt 2009 berücksichtigt werden, und wenn nein, warum nicht? 
Antwort der Parlamentarischen Staatssekretärin Astrid Klug vom 17. September 2008

Ja.

51. Abgeordnete

Welche haushalterischen Auswirkungen wird Angelika die Übertragung der finanziellen VerantworBrunkhorst tung für die Asse vom BMBF auf das BMU (FDP) haben?

\section{Antwort der Parlamentarischen Staatssekretärin Astrid Klug vom 17. September 2008}

Es sollen insbesondere die bisher im BMBF-Haushalt veranschlagten Mittel (Kapitel 3004 Titel 68580 Erläuterungsziffer 11) für die Asse künftig im BMU-Haushalt veranschlagt werden.

Berlin, den 19. September 2008 
Gesamtherstellung: H. Heenemann GmbH \& Co., Buch- und Offsetdruckerei, Bessemerstraße 83-91, 12103 Berlin Vertrieb: Bundesanzeiger Verlagsgesellschaft mbH, Amsterdamer Str. 192, 50735 Köln, Telefon (02 21) 976683 40, Telefax (02 21) 97668344 ISSN $0722-8333$ 